\title{
Seminal Vesicle-secreted Proteins and Their Reactions during Gelation and Liquefaction of Human Semen
}

\author{
Hans Lilja, Jörgen Oldbring," Gunnar Rannevik, ${ }^{*}$ and Cart-Bertil Laurell \\ Departments of Clinical Chemistry and ${ }^{*}$ Urology, University of Lund, Malmö General Hospital, S-214 01 Malmö, Sweden; \\ and ${ }^{\ddagger}$ the Fertility Research Institute, S-217 74 Malmö, Sweden
}

\begin{abstract}
The comparison of measurements of fibronectin and lactoferrin in ejaculates from vasectomized men, subjects with functional deficiency or aplasia of the seminal vesicles, and reference subjects provided evidence that both the fibronectin and the lactoferrin in human seminal fluid originate from the seminal vesicles and the ampullae.

The fibronectin is incorporated in the framework of the seminal gel formed during the immediate postejaculatory phase, whereas the lactoferrin remains in solution. In the seminal gel fibronectin is linked to its predominant structural protein, a high molecular weight seminal vesicle protein (semenogelin). Both the gel-bound fibronectin and semenogelin are progressively fragmented and solubilized by the abundant prostatic kallikreinlike protease (prostate-specific antigen) during and after seminal gel liquefaction. Lactoferrin remains essentially unaffected by the seminal proteases.
\end{abstract}

\section{Introduction}

The minute amount of sperm-dense epididymal fluid mixes with the bulky secretions from the accessory sex glands at ejaculation with a $<10 \%$ admixture of plasma proteins transudated from the intercellular fluids. The bulk of the ejaculate is immediately turned into a viscous gel that normally liquefies within $20 \mathrm{~min}$. The predominant protein in the coagulated part of the ejaculate is a high molecular weight protein, which is also predominant in the secretion from the seminal vesicles (1). This seminal vesicle protein, previously termed HMW-SV protein but coined here as semenogelin, is transformed into three subunits of some 52, 71 , and $76 \mathrm{kD}$ (kilodalton) after reduction (2). The seminal gel liquefies through proteolysis, in parallel with proteolytic fragmentation of semenogelin (3). A series of predominant, basic, low molecular weight proteins in the liquefied ejaculate derive from the fragmentation of semenogelin (2). A prostatic kallikreinlike serine protease $(4,5)$ participates in the liquefaction process. Semenogelin is a physiological substrate of this protease (4) that was first reported as prostate-specific antigen (PSA) ${ }^{1}(6)$.

Fibronectins are adhesive glycoproteins present in a soluble form in extracellular fluids (in blood plasma at a concentration

Address all correspondence to Dr. H. Lilja, Department of Clinical Chemistry, Malmö General Hospital, S-214 01 Malmö, Sweden.

Received for publication 8 December 1986 and in revised form 17 March 1987.

1. Abbreviation used in this paper: PSA, prostate-specific antigen.

J. Clin. Invest.

(C) The American Society for Clinical Investigation, Inc.

$0021-9738 / 87 / 08 / 0281 / 05 \$ 2.00$

Volume 80, August 1987, 281-285 of $0.3 \mathrm{~g} /$ liter) and in less soluble forms on connective tissue cells and on basement membranes. Both forms are composed of two similar but not identical subunits that are joined by an interchain disulfide bond. The subunits have molecular masses of $\sim 225-$ $250 \mathrm{kD}$ (7). Vuento et al. (8) identified immunoreactive fibronectin in seminal fluid by using a rabbit antiserum to blood plasma fibronectin. This fibronectin antigen showed partial antigenic identity with blood plasma fibronectin. Quantitative immunoassay of the fibronectin in seminal fluid gave an average of $1 \mathrm{~g}$ /liter when using blood plasma fibronectin as standard. The albumin concentration, which averages $0.6 \mathrm{~g} /$ liter in seminal plasma (9), may be used as a marker of the transudation of proteins from the intercellular fluids to the accessory sex glands and the male genital ducts. Proteins occurring at higher concentration ratios than the seminal plasma to blood plasma ratio of albumin are actively secreted by the epithelium of the accessory sex glands and their excretory ducts. The similarity in concentration between seminal fluid fibronectin and blood plasma fibronectin excludes the extracellular fluids as major sources of immunoreactive fibronectin in seminal fluid, but the secretory origin of this fibronectin remains to be settled. Vuento et al., who recently succeeded in isolating fibronectin from ejaculated spermatozoa (10), found the sperm-bound fibronectin to consist both of a fraction with a molecular mass similar to that of blood plasma fibronectin and of smaller molecules reacting with antiserum against fibronectin. They drew no conclusions as to whether the variation in immunological reactivity was due to differences in primary structure, complex formation, or proteolysis of fibronectin in semen.

Lactoferrin is another earlier-recognized sperm-coating antigen (11) occurring at a concentration similar to that of fibronectin in seminal fluid. This metal-chelating protein is secreted from phagocytes and various exocrine glands, though usually at lower concentration than in seminal fluid and milk. The seminal vesicles have been suggested to be the major source of lactoferrin in human seminal fluid $(11,12)$.

Our preliminary studies on the electrophoretic distribution of antigens in semen reacting with antiserum to blood plasma fibronectin showed size and charge heterogeneity of the antigen, even though benzamidine was used to retard proteolysis of the seminal proteins (13). We have now followed the reactions of the seminal fibronectin during the early postejaculatory phase and settled the glandular origin of this fibronectin by studies of ejaculates from subjects with anomalies in the accessory sex glands and/or the genital ducts.

\section{Methods}

Reagents. Heparin-Sepharose, Gelatin-Sepharose, and molecular weight markers for polyacrylamide gel electrophoresis (PAGE) were obtained from Pharmacia (Uppsala, Sweden). Monospecific rabbit antisera to human albumin, lactoferrin from milk, and goat anti-rabbit IgG were 
available at the laboratory. Monospecific antisera were raised in rabbits against fibronectin isolated from human blood plasma and against the 5.7-kD basic fragment of semenogelin (2) and PSA isolated from seminal fluid. Antiserum specificity was tested with immunoelectrophoresis and with double immunodiffusion (Ouchterlony).

Patients. Human semen from subjects undergoing investigation for involuntary infertility and from voluntary donors was obtained from the fertility laboratories at Ellenbogen, Malmö and at the Department of Obstetrics and Gynaecology, Malmö General Hospital. Azoospermic semen was obtained from 10 vasectomized subjects. Azoospermic ejaculates were obtained from two subjects with developmental anomalies in the seminal vesicles and in the deferent ducts. In subject A, testes and epididymides were normal in volume and spermatogenesis was normal as verified through biopsy. The right-hand deferent duct was missing and the left-hand duct was thin as judged by physical examination. Neither seminal vesicle was detectable by computer tomography or ultrasonography. The prostatic gland was normal in size. In subject $B$, testes and epididymides volumes were normal. The left-hand deferent duct was missing and the right-hand duct was thin as judged by physical examination. Both seminal vesicles were present but distended and irregular to the touch, whereas the prostatic gland was normal according to computer tomography.

Sample collection. All specimens were obtained by masturbation. Coagulated semen, having a normal liquefaction rate at room temperature, was obtained within 2 min after ejaculation and analyzed immediately as described below. Coagulated semen, washed free of soluble components, was collected and prepared as described earlier (1). Liquefied semen samples (Table I) were brought to the fertility laboratories within $1 \mathrm{~h}$ after ejaculation and the seminal plasma was collected as the supernatant obtained after centrifugation at $800 \mathrm{~g}$ for $15 \mathrm{~min}$. Benzamidine$\mathrm{HCl}$ was added to the seminal plasma to a final concentration of $0.1 \mathrm{M}$ and the samples were then frozen at $-20^{\circ} \mathrm{C}$ until analyzed.

Methods. Sperm count was evaluated under the microscope to confirm azoospermia in semen samples collected from the vasectomized men and from the subjects with defective seminal vesicles. Fructose was determined according to the method of Carvalho and Pogell (14), the detection limit of the assay being $2 \mathrm{mM}$. Zinc was determined with atomic absorption spectrometry. The protein pattern of seminal plasma was analyzed by agarose gel electrophoresis according to a standard procedure (15). PSA, albumin, fibronectin, and lactoferrin were measured by electroimmunoassay (16) that was run in $75 \mathrm{mM}$ barbiturate buffer, pH 8.6, also containing $5 \mathrm{mM}$ of EDTA to counteract interactions with metal ions. A pool made from blood donor plasma was used as standard for the quantitative analysis of fibronectin (concentration of $0.33 \mathrm{~g} /$ liter). The apparent fibronectin concentrations in seminal fluid obtained by the electroimmunoassay were considered to represent the magnitude rather than the absolute concentration of the antigen and were therefore expressed in percent of the plasma pool. Sodium dodecyl sulfate (SDS)-
PAGE in gradients of 70-120 g/liter was run according to Blobel and Dobberstein (17). Immunoblotting after SDS-PAGE was done with the Western blotting technique described by Burnette (18). Immune complexes were detected with horseradish peroxidase-coupled goat anti-rabbit IgG and visualized with 3-amino-9-ethyl-carbazole according to Graham et al. (19).

Protein purification. PSA was purified from pooled seminal plasma as described earlier (4). The concentration of the purified protein was calculated from amino acid analysis after $24 \mathrm{~h}$ hydrolysis in $6 \mathrm{M} \mathrm{HCl}$ at $110^{\circ} \mathrm{C}$ by using the amino acid composition presented by Watt et al. (5). Fibronectin was isolated from fresh-citrated human plasma in a twostep procedure. The fresh-citrated plasma was first applied to a HeparinSepharose column that was equilibrated in Tris buffer at $\mathrm{pH} 7.5$ and then eluted by a $\mathrm{NaCl}$ gradient (20). The major fibronectin peak was localized by electroimmunoassay (16), pooled, and then affinity-purified on a Gelatin-Sepharose column (21). The concentration of the purified protein was calculated assuming a specific absorption $(10 \mathrm{~g} /$ liter, $1 \mathrm{~cm})$ of 12.8 at $280 \mathrm{~nm}$ (22). Lactoferrin was obtained as a by-product during the isolation of other proteins from seminal plasma. The identity of lactoferrin was ascertained by molecular size, charge, and immunoelectrophoresis against a rabbit antiserum to lactoferrin from milk. The concentration of the purified protein was calculated assuming a specific absorption $(10 \mathrm{~g} /$ liter, $1 \mathrm{~cm})$ of 11.2 at $280 \mathrm{~nm}(23)$.

\section{Results}

Glandular origin of seminal fibronectin and lactoferrin. Summarized in Table $I$ are the results of quantitative analyses of 6 different components in azoospermic seminal fluid taken from 2 subjects (A and $B$ ) with defective seminal vesicles; in seminal fluid, from 10 vasectomized men; and in seminal plasma, from 30 reference subjects. The ejaculate volumes of subjects $A$ and $B$ were below the average; though no exact figures were available for those of the vasectomized, in an earlier study we found that on average their ejaculate volumes were $10 \%$ less than those of the reference subjects. Although the average concentrations and ranges of seminal components in ejaculates from vasectomized subjects were similar to those of the reference group, the highest lactoferrin value and the two highest fibronectin values occurred in the smaller group of samples from vasectomized subjects. On electroimmunoassay of seminal plasma fibronectin, the "rockets" were generally more fuzzy in outline than those of fibronectin in human blood plasma. Agarose gel electrophoresis patterns of seminal plasma proteins were within the normal range of variation, both in the ejaculates of vasectomized subjects and in those of reference subjects.

Table I. Comparison of 6 Different Components in the Seminal Plasma from 2 Subjects (A and B) with Functional Deficiency or Aplasia of the Seminal Vesicles, 10 Vasectomized Men, and 30 Reference Subjects with No Signs of Insufficiency of the Accessory Sex Glands

\begin{tabular}{|c|c|c|c|c|c|c|c|}
\hline & Volume & Zinc & Fructose & $P S A$ & Albumin & Lactoferrin & Fibronectin \\
\hline & $m l$ & $m M$ & $m M$ & g/liter & g/liter & g/liter & \% of pool \\
\hline Subject A & 1.4 & 12 & $<2$ & 5.1 & 0.70 & $<0.1$ & $<5$ \\
\hline Subject B & 2.8 & 9.4 & $<2$ & 2.8 & 0.59 & $<0.1$ & $<5$ \\
\hline \multicolumn{8}{|c|}{ Vasectomized men } \\
\hline Mean & ND & 2.1 & 21 & 0.6 & 0.97 & 2.0 & 330 \\
\hline Range & ND & $1.0-6.3$ & $9.0-34$ & $0.30-1.2$ & $0.31-2.2$ & $1.0-4.7$ & $66-650$ \\
\hline \multicolumn{8}{|c|}{ Reference subjects } \\
\hline Mean & 4.3 & 1.9 & 17 & 0.6 & 0.72 & 1.5 & 180 \\
\hline Range & $2.3-7.1$ & $0.36-5.3$ & $8.9-33$ & $0.20-1.8$ & $0.26-2.2$ & $0.90-2.6$ & $44-430$ \\
\hline
\end{tabular}

Seminal plasma was collected as the supernatant obtained after centrifugation at $800 \mathrm{~g}$ for $15 \mathrm{~min}$. 
In the azoospermic samples from subjects A and B the seminal plasma albumin concentration was within the reference range, but their seminal zinc and PSA concentrations were higher than those of any other subject, whereas their fructose, lactoferrin, and fibronectin concentrations were below the sensitivity level of the methods. The electrophoretic protein pattern of ejaculates from subjects A and B denoted predominance of $\alpha_{2-}$ and $\beta_{1}$-migrating proteins and the absence of the normally occurring, cathodally migrating basic fragments of semenogelin.

Reactions of fibronectin and lactoferrin during gelation and liquefaction of semen. We analyzed the apparent molecular mass and the concentration of fibronectin and lactoferrin in the liquid portion of freshly collected ejaculates. The appearance of the basic fragments of semenogelin in the seminal fluid during liquefaction of the ejaculates was evaluated on agarose gel electrophoresis. The liquid portions were recovered from the coagulated ejaculates, starting at $4 \mathrm{~min}$ after ejaculation, as the supernatants obtained after brief centrifugations in a microcentrifuge at 15,000 $g$. The supernatants were immediately analyzed with electroimmunoassay, agarose gel electrophoresis, and SDS-PAGE. In addition, small portions of the separated coagulates, recovered at $4 \mathrm{~min}$ after ejaculation, were also analyzed with SDS-PAGE.

Fig. 1 shows the electroimmunoassay patterns from the timecourse study of fibronectin and lactoferrin in the liquefied portion of a freshly collected ejaculate of a fertile subject. The supernatants recovered by a short centrifugation during the first postejaculatory period contained only trace amounts of fibronectin as long as a gel structure was still present in the ejaculates. The immunoreactive fibronectin rapidly increased to normal seminal plasma values during liquefaction of the gel structure (Fig. 1) at the same time as the cathodally migrating fragments of semenogelin appeared on agarose gel electrophoresis (not shown). There was no apparent decrease in fibronectin concentrations during the first $6 \mathrm{~h}$ after lysis of the seminal gel. Lactoferrin values were unchanged during the same time span studied.

SDS-PAGE followed by immunoblotting with antiserum against the $5.7-\mathrm{kD}$ basic fragment of semenogelin shows the three $50-80-\mathrm{kD}$ subunits of the non-fragmented semenogelin and some low molecular mass fragments of this protein in a reduced sample of seminal coagulate that was washed free of soluble components (lane 2 in Fig. 2 ๑). The presence of fibronectin in this seminal gel structure is demonstrated by the immunoblot shown in Fig. 2 (6). The apparent molecular weight of this gelbound fibronectin, in reduced samples, is not distinctly different from that of fibronectin isolated from human blood plasma (lanes
1 and 3 in Fig. 3). On SDS-PAGE, the predominant part of semenogelin does not have the same molecular mass as fibronectin in non-reduced samples of seminal coagulate (lane $l$ in Fig. 2), and no bands suggesting any covalent complexes between semenogelin and fibronectin in reduced samples of the seminal coagulate can be clearly demonstrated in Fig. 2. Although not convincingly shown in Fig. 2, a minute amount of disulfidelinked complexes or other covalent complexes between fibronectin and semenogelin may be present in the gel structure as judged by the immunoblottings with antisera against fibronectin and the 5.7- $\mathrm{kD}$ basic fragment of semenogelin.

The liquid portions of coagulated ejaculates, collected at 4 min after ejaculation, contained only trace amounts of fragmented fibronectin and semenogelin as evidenced by immunoblottings after SDS-PAGE (not shown). During liquefaction of the gel structure, release of fragmented fibronectin into the seminal fluid was simultaneous with the appearance of the low molecular mass fragments of semenogelin. The immunoblot of fibronectin in Fig. 3 shows the fragmentation pattern of fibronectin in a completely liquefied ejaculate (lane 2 ) and the proteolytic fragmentation of blood plasma fibronectin after exposure of purified fibronectin to purified PSA (lane 4). The enzyme to substrate ratio used in the latter experiment was equal to the molar ratio of PSA to fibronectin in seminal plasma. The fragmentation pattern on SDS-PAGE of fibronectin in seminal fluid during and after liquefaction of the seminal gel was similar to that obtained with purified blood plasma fibronectin exposed to PSA, although the fragments released did not seem to be identical. Seminal lactoferrin appeared essentially undegraded on SDS-PAGE during and after the liquefaction of the gel structure (not shown).

\section{Discussion}

Azoospermic samples were obtained from two subjects with verified anomalies in the deferent and the excretory ducts of the seminal vesicles. The predominance of prostatic secretory components in their ejaculates was verified by the very high concentration of zinc (24) and PSA (25). The lack of secretory components originating from the seminal vesicles and the ampullae was evidenced by subnormal ejaculate volumes, extremely low fructose levels (26), absence of the normal basic fragments of semenogelin on agarose gel electrophoresis (2), and only traces of immunoreactive lactoferrin (11). We conclude that the ejaculates from subjects A and B represent prostatic fluid with a min-
Anti-fibronectin

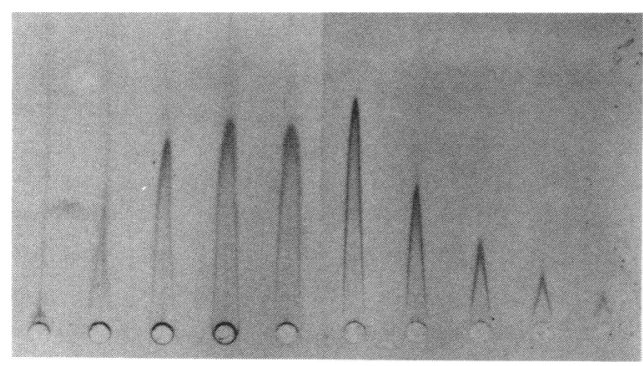

$\begin{array}{llllll}1 & 2 & 3 & 4 & 5 & \text { Standards }\end{array}$
Anti-lactoferrin

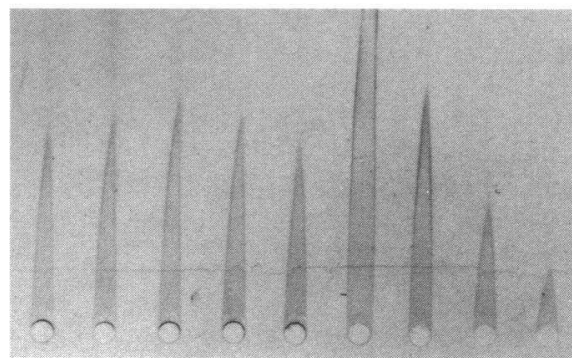

$\begin{array}{llllll}1 & 2 & 3 & 4 & 5 & \text { Standards }\end{array}$
Figure 1. Electroimmunoassay of supernatants recovered, after brief centrifugation in an Eppendorf microcentrifuge (Brinkmann Instruments Inc., Westbury, NY), from a freshly collected ejaculate. The electroimmunoassay of sample 1 started at 4 min, sample 2 at 10 min, sample 3 at $15 \mathrm{~min}$, sample 4 at $60 \mathrm{~min}$, and sample 5 at $6 \mathrm{~h}$ after ejaculation. The left-hand gel contained $20 \mathrm{~g} /$ liter of rabbit antiserum to blood plasma fibronectin, and the righthand gel contained $10 \mathrm{~g} /$ liter of rabbit antiserum to lactoferrin. Pooled

blood donor plasma (fibronectin concentration $0.04-0.66 \mathrm{~g} /$ liter) and purified seminal plasma lactoferrin (0.33-2.6 g/liter) were used as standards. 


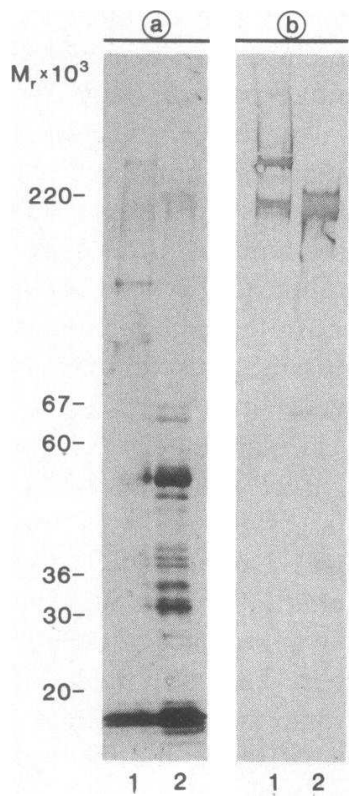

Figure 2. SDS-PAGE in a 70-120-8/ liter polyacrylamide gel gradient. The proteins were immunoblotted after completed electrophoresis against (๑) affinity purified rabbit antibodies to the 5.7-kD basic fragment of semenogelin or (๑) rabbit antiserum to blood plasma fibronectin. (1) Non-reduced seminal coagulate $(1 \mu \mathrm{l})$ and (2) seminal coagulate $(1 \mu \mathrm{l})$ both washed free of soluble components. The sample (2) was reduced (10 mM dithiothreitol [DTT]) and carboxymethylated before electrophoresis.

ute contribution of components derived from the bulbo-urethral (Cowper's) and the urethral (Littre's) glands. Such aplasia of the seminal vesicles has long been known to be connected with no gelation of semen upon ejaculation (26).

The slight differences in ranges and average concentrations in the seminal fluid from vasectomized subjects as compared with those of the reference group showed that no significant amount of fibronectin or lactoferrin in seminal fluid normally originates from the testes and the epididymides. Both from this and the key observation of non-detectable levels of fibronectin and lactoferrin in semen from subjects with functional deficiency or aplasia of the seminal vesicles, we conclude that fibronectin and lactoferrin in seminal fluid quite predominantly originate from the seminal vesicles and the ampullae. Semenogelin, fibronectin, and lactoferrin are the three predominant proteins secreted by these glands. The mass concentration of semenogelin in the seminal vesicle secretion is $\sim 10$ times that of fibronectin and of lactoferrin as judged by the protein pattern on SDS-PAGE of secretion aspirated from the ducts of the seminal vesicles (1).

The analyses of seminal vesicle-secreted proteins during the

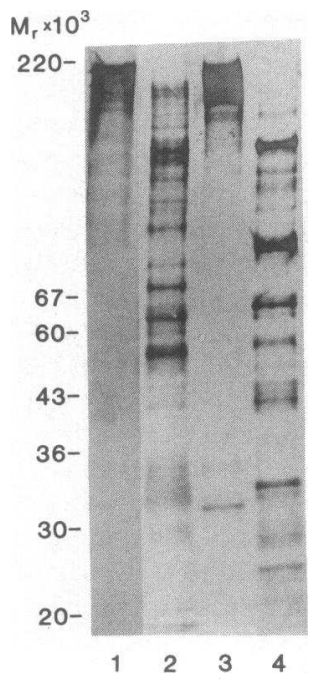

Figure 3. SDS-PAGE in a 70-120-g/liter polyacrylamide gel gradient. All samples were reduced (10 mM DTT) and carboxymethylated pefore electrophoresis. The proteins were immunoblotted after completed electrophoresis against a rabbit antiserum to blood plasma fibronectin. (l) Seminal coagulate $(1 \mu \mathrm{l})$ washed free of soluble components; (2) liquefied ejaculate $(4 \mu \mathrm{l})$ analyzed $2 \mathrm{~h}$ after ejaculation; (3) purified blood plasma fibronectin (1.5 $\mu \mathrm{g})$; and (4) purified blood plasma fibronectin $(1.5 \mu \mathrm{g})$ that was incubated with purified PSA $(4.5 \mu \mathrm{g})$ at $37^{\circ} \mathrm{C}$ and at $\mathrm{pH}$ 7.8 for $2 h$. immediate postejaculatory phase provided evidence that fibronectin and semenogelin are the predominant structural proteins of the seminal gel, whereas lactoferrin remains soluble. Chaistitvanich and Boonsaeng (27) have reported that liquefaction of the seminal gel occurs after the cleavage of disulfide bonds. However, their conclusions were not based on any efforts to simultaneously inhibit the proteolytic activity of PSA. We have found that cleavage of non-covalent bonds was sufficient for the dissolution of the seminal gel structure (1). An absence of covalent bonds between gel-bound semenogelin and fibronectin is in agreement with the results presented here and the fact that neither transglutaminase activity (1) nor immunoreactive Factor XIII (28) has been detected in the human seminal fluid. This implies a polymerizing reaction between fibronectin and semenogelin that is different from the fibronectin interaction with fibrin in the secondary stabilization of the fibrin coagulum by Factor $\mathrm{XIII}_{\mathrm{a}}$. A transglutaminase-mediated covalent linkage occurs as an enhancement reaction of the seminal gel constituents in rodents (29), but the presence of fibronectin has not yet been established in the seminal gel structure of rodents. The results from immunoblottings of both reduced and non-reduced samples of seminal coagulate, washed free of soluble components, did not suggest any major occurrence of disulfide linkages between fibronectin and semenogelin in the gel structure. The immediate conversion of the ejaculate into a gel does not support a catalyzed sulfhydryl-disulfide interchange reaction between semenogelin and fibronectin. Components of the prostatic secretion may induce the polymerizing reaction between fibronectin and semenogelin in a manner similar to that of the fibronectin interaction with platelets and fibrin in the primary haemostasis. The immediate gelation at the ejaculatory mixing of the glandular secretions suggests a stoichometric interaction between some of the components. The high content of positively charged spermine and $\mathrm{Zn}^{2+}$ ions in the prostatic secretion may cause an opening of the fibronectin dimer (30) and the exposure of a molecular surface reactive towards semenogelin. Immediate inactivation of PSA at ejaculation may facilitate the exploration of the nature of the seminal gelation.

The gradual release of immunoreactive fibronectin into the seminal fluid during lysis of the gel structure is apparently the result of progressive cleavage of gel-bound fibronectin. Extensive proteolytic fragmentation of fibronectin was obtained in model experiments when fibronectin isolated from blood plasma was exposed to PSA. Both fibronectin and semenogelin are rapidly degraded by PSA in the seminal fluid. PSA is one of the three most abundant proteins in the human prostatic secretion (4). Our data suggest that the proteolytic activity of PSA is mainly responsible for the fragmentation of the predominant proteins of the seminal gel structure.

There are two major types of the fibronectin family, the hepatic and the fibroblast types (7). The hepatic type circulates freely in blood, whereas inclusion of an extra domain consisting of 90 amino acid residues may account for the decreased solubility of fibronectins made by fibroblasts (31). We were unable to clearly demonstrate any differences in apparent molecular weight between the gel-bound seminal fibronectin and blood plasma fibronectin. Proteolysis of the gel-bound fibronectin may have concealed a possible difference in molecular weight. Functional domains of the fibronectins account for the binding to many different biological compounds such as collagen, heparin, and cell surfaces (7). When the functional domains of the cellular and the hepatic forms of fibronectin are compared, the domains 
are found to be very similar, although minor differences in the sizes of some domains have been noted (32-34). The slight discrepancies between the proteolytic fragmentation of fibronectin in seminal fluid and the fragmentation of blood plasma fibronectin exposed to PSA might suggest a difference between the blood plasma type of fibronectin and the type of fibronectin secreted by the seminal vesicles, although this remains to be established. The hepatic and cellular types of fibronectin both have potential sperm-coating capacity as each contains one common cellular binding domain $(35,36)$. Establishment of the type of fibronectin secreted by the seminal vesicles and the ampullae is a complex task that may be elucidated by a combination of immunochemical and recombinant DNA technology.

\section{Acknowledgments}

We wish to thank Ingrid Wigheden for her expert technical assistance.

This study was supported by grants from the Swedish Medical Research Council (project Nos. B 86-13X-00581-22A and B 87-13X-07903$01 \mathrm{~A})$, the Swedish Society of Medicine, and the Fundàcion Frederico S. A.

\section{References}

1. Lilja, H., and C.-B. Laurell. 1985. The predominant protein in human seminal coagulate. Scand. J. Clin. Lab. Invest. 45:635-641.

2. Lilja, H., C.-B. Laurell, and J.-O. Jeppsson. 1984. Characterization of the predominant basic protein in human seminal plasma, one cleavage product of the major seminal vesicle protein. Scand. J. Clin. Lab. Invest. 44:439-446.

3. Lilja, H., and C.-B. Laurell. 1984. Liquefaction of coagulated human semen. Scand. J. Clin. Lab. Invest. 44:447-452.

4. Lilja, H. 1985. A kallikrein-like serine protease in prostatic fluid cleaves the predominant seminal vesicle protein. J. Clin. Invest. 76:18991903.

5. Watt, K., P.-J. Lee, T. M'Timkulu, W.-P. Chan, and R. Loor. 1986. Human prostate-specific antigen: structural and functional similarity with serine proteases. Proc. Natl. Acad. Sci. USA. 83:3166-3170.

6. Wang, M. C., L. A. Valenzuela, G. P. Murphy, and T. M. Chu. 1979. Purification of a human prostate specific antigen. Invest. Urol. 17: 159-163.

7. Yamada, K. M. 1983. Cell surface interactions with extracellular matrices. Annu. Rev. Biochem. 52:761-799.

8. Vuento, M., E. Salonen, A. Koskimies, and U.-H. Stenman. 1980. High concentrations of fibronectin-like antigens in human seminal plasma. Hoppe-Seyler's Z. Physiol. Chem. 361:1453-1456.

9. Tauber, P. F., J. L. D. Zaneveld, D. Propping, and G. F. B. Schumacher. 1975. Components of human split ejaculates. J. Reprod. Fertil. 43:249-267.

10. Vuento, M., P. Kuusela, M. Virkki, and A. Koskimies. 1984. Characterization of fibronectin on human spermatozoa. Hoppe-Seyler's Z. Physiol. Chem. 365:757-762.

11. Hekman, A., and P. Rümke. 1968. The antigens of human seminal plasma. Protides Biol. Fluids Proc. Colloq. 16:549-552.

12. Roberts, T. K., and B. Boettcher. 1969. Identification of human sperm-coating antigen. J. Reprod. Fertil. 18:347-350.

13. Lilja, H., and H. Weiber. 1983. Synthetic protease inhibitors and post-ejaculatory degradation of human semen proteins. Scand. J. Clin. Lab. Invest. 44:433-438.

14. Carvalho, C. A., and B. M. Pogell. 1957. Modified skatole method for microdetermination of fructose and inulin. Biochim. Biophys. Acta. 26:206.

15. Jeppsson, J.-O., C.-B. Laurell, and B. Franzén. 1979. Agarose electrophoresis. Clin. Chem. 25:629-638.

16. Laurell, C.-B., and E. J. McKay. 1981. Electroimmunoassay. Methods Enzymol. 73:339-369.

17. Blobel, G., and B. Dobberstein. 1975. Transfer of proteins across membranes. J. Cell Biol. 67:835-851.

18. Burnette, W. N. 1981. "Western Blotting": electrophoretic transfer of proteins from sodium dodecyl sulfate-polyacrylamide gels to unmodified nitrocellulose and radiographic detection with antibody and radioiodinated protein A. Anal. Biochem. 112:195-203.

19. Graham, R. C., U. Lundholm, and M. J. Karnowski. 1965. Cytochemical demonstration of peroxidase activity with 3-amino-9-ethylcarbazole. J. Histochem. Cytochem. 13:150-152.

20. McKay, E. J., and C.-B. Laurell. 1980. The interaction of heparin with plasma proteins. J. Lab. Clin. Med. 95:69-80.

21. Richter, H., and H. Horman. 1982. Early and late cathepsin Dderived fragments of fibronectin containing the C-terminal interchain disulfide cross-link. Hoppe-Seyler's Z. Physiol. Chem. 363:351-364.

22. Mosesson, M. V., A. B. Chen, and R. M. Huseby. 1975. The cold insoluble globulin of human plasma: studies of its essential structural features. Biochim. Biophys. Acta. 386:509-524.

23. Masson, P. 1970. La lactoferrine. Thesis. Université Catholique de Louvain, Bruxelles. 232 pp.

24. Mawson, C. A., and M. I. Fischer. 1953. Zinc and carbonic anhydrase in human semen. Biochem. J. 55:696-700.

25. Wang, M. C., M. Kuriyama, L. D. Papsidero, R. M. Loor, L. A. Valenzuela, G. P. Murphy, and T. M. Chu. 1982. Prostate antigen of human cancer patients. Methods Cancer Res. 19:179-197.

26. Amelar, R. D. 1962. Coagulation, liquefaction and viscosity of human semen. J. Urol. 87:187-190.

27. Chaistitvanich, N., and V. Boonsaeng. 1983. Molecular structure of human seminal coagulum: the role of disulfide bonds. Andrologia. 15:446-451.

28. Tauber, P. F., L. J. D. Zaneveld, D. Propping, and G. F. B. Schumacher. 1976. Components of human split ejaculates. J. Reprod. Fertil. 46:165-171.

29. Williams-Ashman, H. G. 1984. Transglutaminases and the clotting of mammalian seminal fluids. Mol. Cell. Biochem. 58:51-61.

30. Vuento, M., T. Vartio, M. Saraste, C. H. von Bonsdorff, and A. Vaheri. 1980. Spontaneous and polyamine-induced formation of filamentous polymers from soluble fibronectin. Eur. J. Biochem. 105:3342.

31. Kornblihtt, A. R., K. Vibe-Pedersen, and F. E. Baralle. 1984. Human fibronectin: molecular cloning evidence for two mRNA species differing by an internal segment coding for a structural domain. $E M B O$ (Eur. Mol. Biol. Organ.) J. 3:221-226.

32. Hayashi, M., and K. M. Yamada. 1981. Differences in domain structures between plasma and cellular fibronectins. J. Biol. Chem. 256: 11292-11300.

33. Sekiguchi, K., A. Siri, L. Zardi, and S. Hakomiri. 1985. Differences in domain structure between human fibronectins isolated from plasma and from culture supernatants of normal and transformed fibroblasts. J. Biol. Chem. 260:5105-5114.

34. Paul, J. I., J. E. Schwarzbauer, J. W. Tamkun, and R. O. Hynes. 1986. Cell-type-specific fibronectin subunits generated by alternative splicing. J. Biol. Chem. 261:12258-12265.

35. Kornblihtt, A. R., K. Umezawa, K., Vibe-Pedersen, and F. E. Baralle. 1985. Primary structure of human fibronectin: differential splicing may generate at least 10 polypeptides from a single gene. EMBO (Eur. Mol. Biol. Organ.) J. 4:1755-1759.

36. Pierschbacher, M. D., and E. Ruoslahti. 1984. Cell attachment activity of fibronectin can be duplicated by small synthetic fragments of the molecule. Nature (Lond.). 309:30-33. 